\title{
Factors Influencing the Status of the Surgical Margin in the Resection of Oral Squamous Cell Carcinoma
}

\author{
KFB Payne* \\ Oral and Maxillofacial Surgery Specialist Registrar, West Midlands Deanery, UK
}

Received: November 15, 2017; Published: December 04, 2017

*Corresponding author: KFB Payne, Oral and Maxillofacial Surgery Specialist Registrar, West Midlands Deanery, UK, Email: karlpayne@doctors.org.uk

Abstract

Aim: Surgical margin status in the resection of oral squamous cell carcinoma (OSCC) is a significant prognostic indicator of recurrence and long term outcome. We sought to investigate the factors (patient, tumour and surgical) at time of surgery that influenced the ability to achieve adequate surgical margins.

Method: We retrospectively reviewed patients who had undergone primary resection of OSCC. Over a 4-year period (2012-2015) 100 patients were surgically treated. Histological derived margins were classified as clear $(\geq 5 \mathrm{~mm})$, close $(<5 \mathrm{~mm})$ or involved (tumour present at resection margin).

Results: Overall, 49\%, 45\% and 6\% had clear, close and involved margins respectively. Of the 100 patients, 28 had stage I, 21 stages II, 7 stages III and 44 stage IV diseases. No relationship was evident between margin status and sex, age $(<65)$, surgical access or individual surgeon. Maximum tumour diameter and depth of invasion were significant factors relating to poorer margins $(p=0.015$ and 0.021$)$. Tumour site appeared to have no impact upon margin status. The histological feature of bone invasion had a significant impact upon poorer margins ( $p=0.015)$, as did a positive node status $(\mathrm{p}=0.0054)$. We were unable to correlate lymphovascular or perineural invasion with margin status.

Discussion: We highlight tumour factors which appear to influence the margin status of resected OSCC, notably tumour size and depth, nodal spread and bone invasion. These all correlate to advanced stage disease being more difficult to treat. Our findings further stress the importance of being able to identify and delineate tumour mass intra-operatively to facilitate a clear resection margin.

Keywords: Head and Neck cancer; Oral squamous cell carcinoma; Surgical margin; Tumour Resection

\section{Introduction}

Recently published UK data stated a 5-year disease-free survival of $74 \%$ and a local recurrence rate of $10 \%$ for patients with surgically treated oral squamous cell carcinoma (OSCC) [1]. Whilst several factors account for poor outcomes within the treatment of OSCC, surgical margin clearances a recognised negative prognostic indicator of local recurrence with mixed evidence of its impact upon long-term survival [2-8]. Current UK guidelines set the goal of $10 \mathrm{~mm}$ as the gold standard for macroscopic margin clearance [9]. Allowing for specimen shrinkage this relates to a $5 \mathrm{~mm}$ pathological margin. In the oral cavity, arguably more so than other sites, this may be difficult or indeed impossible taking into account the need to preserve nearby vital structures.

Furthermore, the ability to compare data on surgical margin significance is marred by the lack of clarity of what defines an 'involved', 'close' and 'clear' margin. The involved margin can either be considered as $<1 \mathrm{~mm}$ [10] or the presence of frank tumour at the resection edge [11]. The widely accepted definition of a close margin is that of $<5 \mathrm{~mm}$ clearance [9]. The importance of the definition of the close and involved surgical margin is paramount when determining the need for adjuvant radiotherapy. With such emphasis placed on the determination of this characteristic and the potential for post-operative morbidity, several authors have argued for $4 \mathrm{~mm}$ [12] or even $3 \mathrm{~mm}$ [8] to be considered a close margin, to better guide adjuvant treatment. Whilst studies have identified factors that influence the adequacy of the surgical margin, including tumour site [2,13], advanced T-stage [2,13,14], tumour size [2,14], depth of invasion [14] and histological features [2], few have drawn a direct comparison of these variables to the adequacy of the resection margin. With this in mind we sought to investigate what factors (patient, tumour and surgical) influence the surgical margin when resetting OSCC and the potential impact this has upon surgical management. 


\section{Method}

Our inclusion criteria were defined as all head and neck cancer patients who had undergone surgical resection for primary oral SCC. From the period 2012-2015, 100 patients met our inclusion criteria and were included within the study. All patients had been seen through a designated head and neck oncology clinic and investigated as appropriate with biopsy and imaging, and MDT discussion. Four Consultant surgeons operated during the 4-year period. The following datasets were collected: demographics (age, sex), clinical (stage, site, surgeon, surgical access) and pathological (margin status, histological features). Histological assessment was performed according to a standardized protocol producing a consistent OSCC dataset, with specimens examined by a Consultant Pathologist.

In line with previously published reports of a similar nature [2] and to allow comparison, we used the following definition of surgical margins: Clear-No evidence of tumour at $5 \mathrm{~mm}$ or greater from the margin, Close-Tumour within $5 \mathrm{~mm}$ of margin, InvolvedEvidence of frank tumour at margin. Univariate analysis was used to compare the aforementioned variables to the three margin groups. Chi-square test was used to analyse contingency tables and the Mann-Whitney U Test was used to compare nominal variables (margin status) to continuous variables (tumour depth). A p-value of $<0.05$ was considered statistically significant.

\section{Results}

Of the 100 patients, 28 had stage I, 21 stages II, 7 stages III and 44 stage IV diseases. Forty-nine patients had clear margins, 45 had close margins and the remaining 6 patients had involved margins. In all involved cases the deep margin was the site of involvement. With regard close margins, $40 \%$ (18) were close on the deep margin, $42.2 \%$ (19) on the lateral/mucosal margin and $17.8 \%$ (8) close on both margins. The study cohort consisted of 54 male and 46 female patients. For the purpose of categorising age, a cut off of 65 was used, with 61 patient's $\geq 65$ years of age. No significant correlation was found between margin status and gender or age.

The most commonly used surgical approach was per oral (86\%), with lip split (2\%), Weber-Ferguson (4\%) and Transcervical (8\%) approaches also utilised. No association was found between surgical access and margin status. Furthermore, no significant difference was found between operating surgeon and margin status. Seventy-three patients underwent micro vascular reconstruction and 74 patients received modified radical neck dissection. There was no significant difference in surgical margin status between the micro vascular and non- micro vascular cohorts. For the purposes of data continuity, when no neck dissection was performed in the clinically node negative neck then nodal metastasis and extracapsular spread was recorded as not present (N0). Thirty-five percent of patients received post-operative radiotherapy.

With regard tumour site, the majority were tongue cancers (45\%), followed by cancers of the alveolus (21\%). No association was found between site and margins status. Whilst tongue cancers exhibited the majority of involved margins, this was likely skewed due to higher patient numbers in this group. Tumours of the lower alveolus appeared to have proportionately more close margins and all palatal tumours were excised with close margins. Tumours of a greater maximum diameter exhibited poorer margins $(\mathrm{p}=0.015)$, with the majority lying in the T2 category of $21-40 \mathrm{~mm}$ and almost all of those $>40 \mathrm{~mm}$ in size demonstrating close or involved margins. Average depth of invasion for clear vs. close/involved margins was $7.5 \mathrm{~mm}$ and $11.7 \mathrm{~mm}$ respectively. To evaluate tumour depth, as no clear validated categories are available, the Mann Whitney U test was used to compare clear vs. close/involved margins to depth of invasion on a continuous scale. A greater depth of invasion was significantly associated with close/involved margins $(\mathrm{p}=0.021)$. When evaluating nodal status, there was a significant relationship between patients with nodal metastases and extra capsular spread, and inadequate margins $(\mathrm{p}=0.014)$.

Histopathological data revealed a significant relationship between bone invasion and inadequate margins $(p=0.015)$. There was no significant association between margin status and the other histological variables of lymphovascular invasion, perineural invasion, severe dysplasia, and non-cohesive growth front and tumour differentiation. Using descriptive analysis, tumours with perineural and/or lymphovascular invasion had a greater proportion of close margins compared to other histological variables. The majority of tumours appeared to have a moderately differentiated non-cohesive invasive tumour front (75\% and $86 \%$ ), which may explain why the low numbers in other categories negated any clear trend in these variables towards margin status.

\section{Discussion}

If the adequacy of the resection margin for OSCC is an indicator of outcome and need for adjuvant therapy, then the aim of this study was to identify what factors, present at the time of surgery, both impact and are within our control to influence the quality of our resection. Our involved margin status of $6 \%$ is in line with previously reported figures (4.5-22\%), but forming a direct comparison is difficult. Whilst those studies that use the definition of an involved margin as tumour at the resection have an average of approximately $5-10 \%$, those that state $<1 \mathrm{~mm}$ as involved exhibit skewed data with figures as high as $24 \%$ [6]. Perhaps for this reason, recent research has focused on the definition of the close surgical margin, as this is seen as the 'grey' area of deciding whether adjuvant treatment is required $[5,8]$.

It was our hypothesis that we would discover that larger tumours and those more posterior in the mouth would have a higher frequency of close or involved margins, due in part to difficulty in surgical access and resection. However, from our data, tumour site does not appear to influence adequacy of resection margin. In a series of 110 patients, Lawaetz et al. reported that floor of mouth cancers had significantly poorer margins when compared to tongue cancers [13] and this finding was mirrored by Nason et al. who found that in 277 patients, tongue cancers had significantly clearer margins than other sites [8]. The ability to manoeuvre the tongue within the mouth when compared to other 'fixed' anatomical sites potentially explains these previous findings. Like Sutton et al., we 
were unable to find this correlation between site and margin status [2], perhaps due to a disproportionately large number of tongue tumours compared to other sites.

Evaluating surgical factors, the surgeon and complexity of the procedure had no impact upon the margin status. The majority of our resections were performed via oral access, which leaves us unable to fully analyse the impact of more complex surgical approaches. Within our cohort, tumours of increasing diameter and depth of invasion had significantly poorer margins ( $p=0.015$ and 0.021), supporting the findings of Sutton et al. and Girardi et al. We assume these relationships relate to difficulty in surgical resection, whereby either the surgeon identified the tumour margin and chose to perform a close resection to preserve nearby vital structures, or was unable to accurately assess the tumour margin in the first instance and performed a normal wide excision, as may be the case with a deep irregular infiltrating tumour. As in other studies [2], the presence of nodal metastases was significantly correlated to poorer margins $(\mathrm{p}=0.0054)$, with 5 of the 6 patients with involved margins demonstrating nodal metastases.

However, the presence of extra capsular spread did not appear to have a correlation to margin status. The above findings draw the conclusion that tumours with increased aggression and metastatic potential demonstrated poorer surgical margins. These findings are further highlighted when comparing overall stage of disease to margin status. Those with early stage I and II disease had a significant trend towards clearer margins when compared to late stage III and IV disease $(\mathrm{p}=0.02)$. Whilst previous research has reported a strong link between inadequate margins and the features of the invasive tumour front and perineural and vascular invasion [2], like Lawaetz et al. and Girardi et al. we were unable to replicate these findings $[13,14]$. Of all the histological characteristics we analysed, the presence of bone invasion was the only variable significantly associated with inadequate margin status.

In resections of large tumours infiltrating bone, the desire is undoubtedly to preserve as much hard tissue as possible to broaden reconstructive options, which may explain this finding. Sutton et al. noted that given the correlation between pathological findings and margin status, then resection clearance "should be regarded as a product of aggressive tumour behaviour in addition to, or even rather than, inadequate surgical resection". Their suggestion was the potential use of larger initial biopsies, to better identify those pathological characteristics and prepare the surgeon for a wider excision. This notion is obviously balanced against the surgical morbidity in resection and reconstruction and the desire to preserve vital structures. One option is the use of intra-operative frozen sections, but its clinical application is contentious for reasons of cost and reliability [15].

If our findings are applied to clinical practice, the conclusion is that the only parameters we are able reliably to use to predict margin status are those of the already adoptedpre-operative staging assessment of tumour size, depth of invasion and nodal metastasis. McMahon et al. discussed the importance of using an 'anatomical approach' in the resection of OSCC [16]. When comparing surgical margin adequacy between 2 cohorts a decade apart, they demonstrated that the use of modern imaging and in particular post-acquisition processing to better define tumours reduced the involved margin rate from $37 \%$ to $5 \%$. A similar approach was taken by Ota et al., who used a combination of US, CT and MRI to evaluate buccal SCC cancers and define them preoperatively into 3 categories based upon depth of invasion in relation to the buccinators muscle [17]. They reported increased local control and long term survival when compared to previous surgical methods. As the deep aspect was responsible in all of our involved margins, and over half of the close margins, such a technique may be applicable to other sites in the mouth. Whilst the above enhanced pre-operative assessment can improve outcomes, the ability of the operating surgeon to better visualise and delineate the tumour mass intra-operatively is the gold standard.

To these effect agents such as Toluidine blue [18] and Lugol's Iodine [19] have been suggested, with apparent positive outcomes. The use of Lugol's iodine is currently the topic of a UK based multicentre RCT [20]. Perhaps the most exciting advancement is the use of 3D navigation to improve tumour resection rates. Using this technology the surgeon is able to define the tumour on preoperative imaging and set a tumour "distance wall" correlating to a safe resection margin in relation to nearby vital structures, this is then used intra-operatively to guide the exact resection [21]. This dataset represents the beginning of an evolving head and neck database within our unit. As such we accept that the most significant limitation of our study is the absence of any recurrence or long term survival data, and relatively low patient numbers.

\section{Conclusion}

If we accept that surgical margin status is a significant negative prognostic indicator of outcome in the treatment of OSCC, then efforts should continue to better identify factors relating to and methods to improve the standard of resected margins. Our findings add weight to the evidence that the characteristics of tumour invasion to deep tissues and bone together with nodal metastases correlate to the adequacy of surgical margins, regardless of tumour site or surgical procedure complexity. It remains to be seen how we can better improve surgical margins by delineating tumour mass intra-operatively.

Word count $=2,490$

\section{References}

1. Rogers SN, Brown JS, Woolgar JA, Lowe D, Magennis P, et al. (2008) Survivial following primary surgery for oral cancer. Oral Oncology 45: 201-211.

2. Sutton DN, Brown JS, Rogers SN, Vaughan ED, Woolgar JA (2003) The prognostic implications of the surgical margin in oral squamous cell carcinoma. International Journal of Oral and Maxillofacial Surgery 32: 30-34.

3. Cook JA, Jones AS, Phillips DE, Soler-Lluch E (1993) Implications of tumour in resection margins following surgical treatment of squamous cell carcinoma of the head and neck. Clinical Otolaryngology and Applied Sciences 18(1): 37-41. 
4. Larsen SR, Johansen J, Sorensen JA, Krogdahl A (2009) The prognostic significance of histological features in oral squamous cell carcinoma. Journal of Oral Pathology and Medicine 38: 657-662.

5. Wong LS, McMahon J, Devine J, McLellan S, Thompson E, et al. (2008) Influence of close resection margins on local recurrence and diseasespecific survival in oral and oropharyngeal carcinoma. British Jounral of Oral and Maxillofacial Surgery 50: 102-108.

6. Eldeeb H, Macmillan C, Elwell C, Hammod A (2012) The effect of the surgical margins on the outcome of patients with head and neck squamous cell carcinoma: as single institution experience. Cancer Biolology \& Medicine 9(1): 29-33.

7. Kurita H, Nakanishi Y, Nishizawa R, Xiao T, Kamata T, et al. (2010) Impact of different sirgical margin conditions on local recurrence of oral squamous cell carcinoma. Oral Oncology 46: 814-817.

8. Nason R, Binahmed A, Pathak KA, Abdoh AA, Sandor GKB (2009) What is the adequate margin of surgical resection in oral cancer? Oral Surg Oral Med Oral Pathol Oral Radiol Endod 107: 625-629.

9. BAHNO (2011) Head and Neck Cancer, Multidisciplinary Management Guidelines. The Royal College of Surgeons of England, England, pp. 1-382.

10. Woolgar JA (2006) Histopathological prognostication in oral and oropharyngeal squamous cell carcinoma. Oral Oncology 42: 229-239.

11. Batsakis JG (1999) Surgical excision margins: a pathologist's perspective. Advances in Anatomic Pathology 6: 140-148.

12. Alicandri Ciufelli M, Bonali M, Piccinini A, Marra L, Ghidini A, et al. (2013) Surgical margins in hea dand neck squamous cell carcinoma: what is close? Eur Arch Otorhinolaryngol 270(10): 2603-2609.

13. Lawaetz M, Homoe P (2014) Risk factors for and consequences of inadeqaute surgical margins in oral squamous cell carcinoma. Oral Surg Oral Med Oral Pathol Oral Radiol 118: 642-646.
14. Girardi FB, Zanella VG, Kroef RG (2013) Correlation between clinical and pathological data and surgical margins in patients with squamous cell carcinoma of the oral cavity. Braz J Otorhinolaryngol. 79(2): 190-195.

15. Di Nardo LJ, Lin J, Karageorge LS, Powers CN (2000) Accuracy, utility, and cost of frozen section margins in head and neck cancer surgery. Laryngoscope 110(10): 1773-1776.

16. McMahon JD, Devine JC, Hetherington J, Bryson G, McLellan D, et al. (2011) Invovled surgical margins in oral and oropharyngeal carcinoma an anatomical problem? British Journal of Oral and Maxillofacial Surgery 49: 172-175.

17. Ota Y, Aoki T, Karakida K, Otsuru M, Kurabayashi H, et al. (2009) Determination of deep surgical margin based on anatomical architecture for local control of squamous cell carcinoma of the buccal mucosa. Oral Oncology 45: 605-609.

18. Allegra E, Lombardo N, Puzzo L, Garozzo A (2009) The usefulness of toluidine ataining as a diagnostic tool for precancerous and cancerous oropharyngeal and oral cavity lesions. Acta Otorhinolaryngol Ital 29(4): 187-190.

19. Petruzzi M, Lucchese A, Baldoni E, Grassi FR, Serpico R (2010) Use of Lugol's iodine in oral cancer diagnosis: An overview. Oral Oncology 46: 811-813.

20. McCaul J, Sutton D, Shaw R, Mehanna H, Hislop S, et al. (2015) LIHNCS (Lugol's Iodine in Head and Neck Cancer Surgery): a UK Multicentre Prospective Randomised Controlled Trial. Report of primary and secondary outcome measures. British Journal of Oral and Maxillofacial Surgery 53: e112.

21. Rana M, Essig H, Eckardt AM, Tavassol F, Ruecker M, et al. (2012) Advances and Innovations in Computer-Assisted Head and Neck Oncologic Surgery. J Craniofac Surg 23: 272-278.

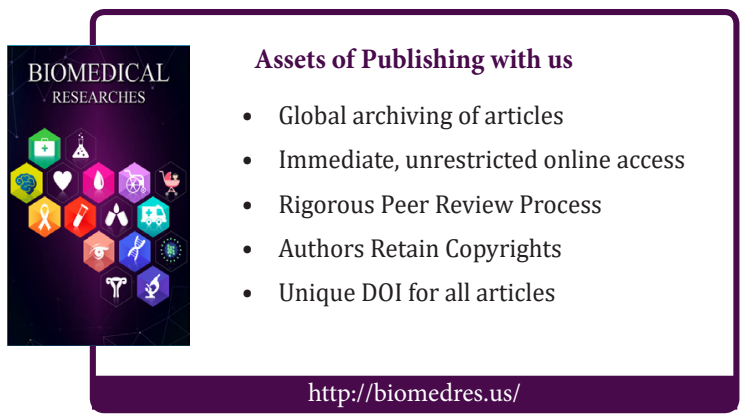

